REGARDS

SUR LEECONOMIE ALLEMAND

BULLETIN ECONOMIQUE DU CIRAC
Regards sur l'économie allemande

Bulletin économique du CIRAC

$67 \mid 2004$

Varia

\title{
Le phénomène discount en Allemagne
}

Caroline Guiot

\section{OpenEdition}

\section{Journals}

Édition électronique

URL : http://journals.openedition.org/rea/3804

DOI : 10.4000/rea.3804

ISBN : 978-2-8218-0830-0

ISSN : 1965-0787

Éditeur

CIRAC

Édition imprimée

Date de publication : 1 juillet 2004

Pagination : 27-32

ISSN : 1156-8992

Référence électronique

Caroline Guiot, « Le phénomène discount en Allemagne », Regards sur l'économie allemande [En ligne], 67 | juillet 2004, mis en ligne le 08 octobre 2009, consulté le 14 novembre 2019. URL : http:// journals.openedition.org/rea/3804; DOI : 10.4000/rea.3804 


\section{Le phénomène discount en Allemagne}

\section{Caroline Guiot}

L'Allemagne est considérée comme le fief du discount alimentaire. Plus qu'ailleurs, la composante prix y est décisive dans la décision d'achat. Une étude de l'institut allemand de la consommation GfK, publiée en mars 2004, portant sur la sensibilité au prix des consommateurs de six pays européens, montre que pour $56 \%$ des Allemands, en 2003 , le facteur prix est déterminant dans le processus d'achat. Un taux plus élevé que la moyenne européenne qui s'établit à 49\%. Et ce taux est stable depuis 2001, alors que dans l'ensemble des pays sondés, la sensibilité au prix diminue (hormis en Italie). Or, bien qu'importante, cette sensibilité n'explique pas à elle seule le succès fulgurant du discount alimentaire en Allemagne. La conjoncture économique, le passage à l'euro, et surtout un concept et des atouts maîtrisés de la part des discounters ont accentué les comportements des consommateurs allemands en matière d'achats alimentaires.

En France, le débat sur l'inflation consécutive au passage à l'euro ressentie par les consommateurs, par opposition aux mesures économiques et institutionnelles de l'INSEE, est récent. En Allemagne, par contre, il est né immédiatement après le basculement. D'ailleurs, la crainte du renchérissement des prix suscité par l'euro a été exploitée par les distributeurs alimentaires dès la fin 2001, notamment avec une forte campagne publicitaire de l'enseigne Aldi, affirmant : « chez nous, aucun produit ne sera plus cher en passant à l'euro » (Bei uns wird durch den Euro kein einziges Produkt teurer). Cette campagne visait doublement à fidéliser une clientèle réduisant ses dépenses alimentaires et à conforter les positions du discount dans un paysage de la distribution alimentaire en pleine restructuration.

Dans le contexte économique difficile de la fin des années 90, et après l'euphorie éphémère du record de croissance de l'an 2000, le basculement à l'euro au $1^{\text {er }}$ janvier 2002 avait eu une répercussion véritablement négative sur les dépenses de consommation alimentaire, d'autant qu'il coïncidait avec un fort renchérissement des produits frais lié aux récentes épizooties (crise de la « vache folle ») et aléas météorologiques européens. Le chiffre d'affaires global du commerce alimentaire de détail, qui était en constante croissance depuis 1995 (+8,8 \% en six ans), est passé de 133,5 milliards $€$ en 2001 à 132,2 milliards $€$ en 2002, soit une diminution de $0,97 \%$. Et, selon les estimations de l'institut GfK, il aura baissé encore de 0,53\% en 2003.

Or la réduction des achats alimentaires des ménages ne touche pas tous les acteurs de la branche. Si les supermarchés la subissent de plein fouet, perdant 3,7 points de parts de marché entre 2001 et 2002 (de 39,8 \% à 36,1\%), les magasins discount sont les grands gagnants de cette redistribution des dépenses alimentaires des ménages. En effet, la part de marché des discounters passait de $33,6 \%$ à $37,7 \%$, soit une augmentation de 5 millions $€$ du chiffre d'affaires réalisé par les enseignes Aldi, Lidl et autres en un an seulement.

Toutefois, dans ce contexte de concurrence accrue, et pour relancer la consommation des ménages, l'ensemble des commerces a voulu jouer la carte du discount. Ainsi est apparu le slogan « Radin, c'est chic! » (Geiz ist geil !). Bien

Un positionnement qui se généralise 
que lancé hors de la branche du commerce alimentaire de détail, il a influencé toutes les politiques de prix dans le secteur depuis 2002.

Parts de marché des distributeurs alimentaires en RFA (en \%)

\begin{tabular}{lrrrrr|}
\hline & $\mathbf{1 9 9 8}$ & $\mathbf{1 9 9 9}$ & $\mathbf{2 0 0 0}$ & $\mathbf{2 0 0 1}$ & $\mathbf{2 0 0 2}$ \\
Discounters & 29,8 & 30,7 & 32,1 & 33,6 & 37,7 \\
Supermarchés (surface > $\mathbf{8 0 0} \mathbf{~ m 2 )}$ & 40,2 & 40,6 & 39,8 & 39,8 & 36,1 \\
Commerces alimentaires traditionnels (surface < 799 m2) & 23,3 & 21,6 & 20,6 & 18,8 & 18,3 \\
Autres commerces alimentaires de détail & 6,7 & 7,1 & 7,5 & 7,9 & 7,9 \\
\hline
\end{tabular}

Source des données : IRI / GfK Panel Services Consumer Research GmbH. NB : Les hypermarchés sont peu présents en Allemagne.

Beaucoup d'entreprises ont misé sur des remises et des campagnes de promotions dans le but affiché de ramener les consommateurs dans les magasins. Mais c'est l'effet inverse qui s'est produit : non seulement, cela n'a pas redressé le moral des consommateurs, ces slogans soulignant la nécessité d'épargner, mais surtout ces politiques ont mis un certain nombre d'entreprises dans des situations financières encore plus difficiles, entraînant un nombre croissant de dépôts de bilan. A l'opposé, les discounters, pour lesquels les bas prix sont le premier principe, ont pu s'appuyer sur un positionnement clair et sur un véritable concept, développé grâce à la maîtrise de leurs atouts.

\section{Les principaux acteurs du discount alimentaire en Allemagne}

-ALDI. Créé en 1948 par les frères Albrecht, le groupe est scindé aujourd'hui en deux zones géographiques, gérée chacune par l'un des deux frères : Aldi Nord (Aldi-Einkauf GmbH \& Co. oHG, Essen ; www.aldi-essen.de) et Aldi Süd (Aldi-Einkauf GmbH \& Co. oHG, Mülheim an der Ruhr ; www.aldi-sued.de).

Aldi Süd (plus de 2200 filiales) est implanté en Australie depuis 2001, après avoir conquis le sud de l'Allemagne, l'Autriche, les USA, l'Angleterre et l'Irlande. Aldi Nord (plus de 1260 filiales) s'exporte dans le voisinage: nouveaux Länder, Hollande, Belgique, Danemark. France, Espagne. Un certain secret entoure l'empire des frères Albrecht. Outre le fait que le statut juridique adopté ne leur impose pas de publier de rapport annuel, ils ne communiquent leurs données que parcimonieusement. CA 2001 : Aldi Nord : 11,6 milliards € ; Aldi Süd : 9,9 milliards €.

- Lidl. Création en 1973 de la Lidl Stiftung \& Co. KG, Neckarsulm (www.lidl.de).

Avec plus de 3000 filiales, Lidl est présent en Allemagne, France, Belgique, Hollande, GrandeBretagne, Italie, Espagne et au Portugal. Lidl est également réputé pour ne pas divulguer ses données. CA $2001: 6,26$ milliards $€$.

- Plus (groupe Tengelmann), création en 1972 : Plus Warenhandelsgesellschaft mbH, Mülheim an der Ruhr (www.shop.plus.de).

Les quelque 2700 filiales de Plus se situent essentiellement en RFA. CA 2001 : 5,3 milliards $€$.

- Penny (groupe Rewe), enseigne créée au début des années 70 : Penny Markt GmbH, Cologne (www.penny.de)

Les quelque 2400 filiales de Penny se situent principalement en Allemagne. CA 2001 : environ 5 milliards $€$.

Les facteurs clés de succès des discounters

La logistique : pierre angulaire du système
Derrière une enseigne discount, plusieurs facteurs clés de succès sont mis en œuvre. La simplicité est la règle d'or, tant pour le consommateur (peu de choix) que pour le distributeur (peu de contraintes) ; elle s'accompagne d'une compression systématique des coûts. Les choix minimalistes dans la présentation des magasins, où les palettes font office de gondoles, permettent de réduire au minimum les charges liées au personnel. Enfin, LE principe clé : la gamme de produits restreinte. Alors que les hypermarchés français comptent en moyenne 100000 références, Aldi par exemple n'en fournit que 600, concentrées sur les aliments secs et les liquides, et récemment quelques produits surgelés et réfrigérés : ce qui était une contrainte dans les années d'après-guerre a été transformé en force.

Cette force se démultiplie en trois leviers: 1$)$ une rotation très rapide des stocks : donc des approvisionnements réguliers et peu de pertes ; 2 ) un approvisionnement effectué à l'échelle mondiale sur des quantités très importantes, ce qui signifie un fort pouvoir de négociation sur les prix (les achats d'Aldi repré- 
sentent 2 milliards € par an) ; 3) une logistique adaptée aux gros volumes, diminuant les coûts de transport. Ainsi d'une part, les relations privilégiées avec leurs fournisseurs permettent aux discounters de négocier beaucoup, tant sur les prix que sur la qualité des produits : un fournisseur qui faillit au cahier des charges très strict (quantité, qualité, fraîcheur des produits, prix) est sanctionné par la perte de contrats d'approvisionnements. D'autre part, la logistique occupe une place primordiale. Bien que les achats soient centralisés, l'existence de plateformes d'approvisionnement régionalisées des magasins permet d'optimiser la gestion des produits à forte rotation et du transport.

En définitive, l'objectif final est bien de proposer les prix les plus bas du marché pour l'ensemble des références, et surtout des prix stables sur le long terme, hors promotions et offres spéciales, afin d'être toujours attractifs. Le dernier principe consiste, malgré le succès (croissance du chiffre d'affaires, augmentation du nombre de ménages achetant dans l'enseigne), à rester économe, à conserver le positionnement discount, à ne pas changer les règles d'or fixées initialement : simplicité de présentation, assortiment restreint, prix bas.

\section{Un concept ancré dans l'histoire et les valeurs de la société allemande}

Le concept de ce que, aujourd'hui, on appelle le discount est né dans l'immédiat après-guerre. Le premier magasin Aldi, à l'époque une épicerie de quartier, ouvrait en 1946 à Essen, dans la Ruhr. Gamme réduite et prix bas reflétaient alors la pénurie de produits alimentaires qui sévissait dans un pays en ruines. Avec le regain économique qui a suivi l'introduction du mark en 1948, l'entreprise s'est développée, portée en outre par son concept adapté à une clientèle ouvrière. Si le «miracle économique » a ensuite permis à l'entreprise de prospérer dans l'Allemagne entière, Aldi doit également sa croissance à des facteurs socio-culturels, son concept correspondant aux valeurs de la génération de la guerre qui appelait de ses vœux « une prospérité modeste, mais croissante » (Appel/Schipperges, 2002).

Les concurrents d'Aldi ne sont apparus qu'au début des années 70, mettant à profit l'émergence de l'ascétisme postmatérialiste d'après 1968. Ces années marquent l'essor à grande échelle du phénomène discount outre-Rhin. Depuis, le recrutement de la clientèle s'est diversifié, s'étendant pratiquement à l'ensemble de la société. Le discount séduit aujourd'hui une partie des jeunes générations, à la fois hédonistes et anticonformistes, comme des plus anciennes qui, se sentant désorientées par les mutations, se replient sur la nostalgie. Quant aux baby boomers d'après guerre, aujourd'hui quinquagénaires et plus, ils cultivent la fidélité à leur fonds idéologique protestataire, mais en y associant une nouvelle valeur : la consommation plaisir, à laquelle ils s'adonnent avec d'autant moins de honte qu'ils se donnent les moyens d'acheter le superflu en réduisant leurs dépenses sur l'indispensable. Les bas prix n'expliquent pas seuls le succès du discount en Allemagne ; c'est aussi un phénomène de société.

Le discount donne lieu aujourd'hui à un véritable phénomène de mode. Les sites Internet fleurissent : « Fan d'Aldi » (aldifan.de), « Avare » (geizkragen.de), «Acheter, mais où ? » (kaufenaberwo.de), autant de lieux d'échanges entre consommateurs, de comparaison des promotions d'une enseigne ou d'une autre, de partage des 'bons plans'. II est vrai que la culture Internet est particulièrement propice à la transparence des prix et à la chasse aux bonnes affaires, dans l'alimentaire comme ailleurs.

Ces sites jouxtent ceux, officiels, des discounters. Or par rapport à la France, une différence essentielle de présentation des sites Internet des enseignes apparaît : alors que ceux des grandes enseignes de distribution alimentaire françaises présentent essentiellement leurs marques, leurs valeurs (commerce équitable, développement durable,...), leurs axes de développement, leurs comptes, des conseils (diététiques ou autres), les sites des discounters allemands proposent uniquement leurs offres promotionnelles du moment, les adresses des magasins et quelques offres d'emploi, le tout traduit en autant de langues que de pays dans lesquels ils sont implantés. Nous touchons là encore à des tendances de comportements distinctes: les Allemands sont principalement axés sur le prix des produits (du moins dans l'alimentaire), alors que, pour les Français, la qualité des produits et les valeurs associées à l'enseigne ou à la marque sont déterminantes dans le processus d'achat.
Règle d'or : conserver le positionnement discount

Un phénomène de mode, relayé par Internet

Sites des enseignes allemandes : priorité aux bas prix 
Stagnation du commerce en ligne

Les produits frais échappent encore au discount

Parallèlement, il est intéressant de noter que la vente de produits alimentaires sur Internet, quelles que soient les enseignes, ne s'est guère développée en Allemagne, pas plus qu'en France, malgré la publicité faite autour du lancement des Ooshop.fr et autre Telemarket.fr. Cela s'explique en partie par les critères sensoriels mobilisés lors de l'achat des biens alimentaires, qui ne peuvent se retrouver sur ordinateur. Au-delà, cela s'explique par la nécessité d'un équipement informatique important des ménages (plus prononcé pourtant en Allemagne qu'en France) et, surtout, par la logistique extrêmement coûteuse à mettre en place pour effectuer les livraisons, donc la faible rentabilité de cette offre de services par les enseignes - dans les deux pays.

Pour consolider leur nouvelle position de leader du commerce alimentaire de détail, les discounters suivent plusieurs axes d'innovation: l'extension de la gamme de produits proposée, l'exploitation des marques distributeurs et le déploiement géographique. Malgré la supériorité des magasins discount et l'importance des supermarchés, une part non négligeable de la vente de certains aliments est encore réalisée par les commerces spécialisés comme pour le pain et la viande fraîche par exemple, ou sur les marchés pour les œufs et les pommes de terre.

Lieu d'achat par type de produits en 2003 (répartition en \%*)

\begin{tabular}{|c|c|c|c|c|c|c|}
\hline & Discounters & Supermarchés & $\begin{array}{l}\text { Commerce } \\
\text { spécialisé }\end{array}$ & $\begin{array}{l}\text { Commerce alim. } \\
\left(\text { surface }<800 \mathrm{~m}^{2}\right)\end{array}$ & Marchés & Autres \\
\hline Viande et charcuteries & $40 \%$ & $27 \%$ & $19 \%$ & $10 \%$ & $2 \%$ & $2 \%$ \\
\hline CEufs & $43 \%$ & $20 \%$ & $3 \%$ & $7 \%$ & $21 \%$ & $6 \%$ \\
\hline Pommes de terre & $38 \%$ & $27 \%$ & $2 \%$ & $9 \%$ & $18 \%$ & $6 \%$ \\
\hline Pain & $32 \%$ & $16 \%$ & $43 \%$ & $6 \%$ & $1 \%$ & $2 \%$ \\
\hline Lait & $53 \%$ & $32 \%$ & $0 \%$ & $12 \%$ & $2 \%$ & $1 \%$ \\
\hline Fruits en conserve & $60 \%$ & $30 \%$ & $0 \%$ & $9 \%$ & $0 \%$ & $1 \%$ \\
\hline Sucre & $53 \%$ & $34 \%$ & $0 \%$ & $11 \%$ & $0 \%$ & $2 \%$ \\
\hline
\end{tabular}

Source des données : ZMP, Nachfrage privater Haushalte nach Nahrungsmitteln 2003. *) Estimations.

Mais le discount développe ce segment
L'exploitation des marques distributeurs
Faut-il y voir des niches réservées aux commerces spécialisés, inaccessibles aux discounters ? Pas du tout. En plus de la viande surgelée, proposée dès les années 80 grâce à la maîtrise du grand froid, Aldi s'est attaqué dès 2002 à la vente de viande en libre-service dans les rayons. Et les autres discounters ont suivi cette voie. Les consommateurs approuvent : l'achat de viande fraîche en libre service est considéré comme plus facile et plus rapide. Le choix entre les discounters et les autres magasins se fait donc sur le prix et sur le packaging (grosses quantités versus achats d'appoint). De la même façon, en 2001, le groupe Metro a lancé le concept de vente de pain en libre-service à prix discount : la chaîne Backwerk est née, utilisant les mêmes recettes que les discounters d'alimentation générale (assortiment restreint, coûts de personnel réduit, produits en libre-service). L'exemple a essaimé car, depuis, d'autres enseignes (Brödis, Baking-Friends) se sont créées, avec un certain succès.

Importance du chiffre d'affaires réalisé par la vente des marques de distributeurs

\begin{tabular}{|lcc|}
\hline 2002, en milliards $€$ & CA total & CA des marques de distributeurs \\
ALDI & 24,6 & 16,0 \\
Autres discounters & 24,5 & 8,0 \\
Autres commerces alimentaires de détail & 83,1 & 5,5 \\
\hline
\end{tabular}

Source des données : GfK Panel Services Consumer Research GmbH. Enquête auprès de 12.000 ménages allemands.

Un autre point fort exploité activement par les discounters est la vente des marques de distributeurs. En 2002, ces produits sans étiquette représentent $65 \%$ du chiffre d'affaires réalisé par Aldi, contre à peine 6,6\% du chiffre d'affaires des autres commerces alimentaires de détail. Dans l'offre des discounters, les produits sans nom ou à celui du distributeur constituent des alterna- 
tives aux marques connues, nécessairement plus chères, valorisant ainsi la politique de bas prix affichée, et offrant une tribune publicitaire gratuite à l'enseigne. Les consommateurs, loin de souligner une différence qualitative des produits de distributeurs, les plébiscitent, notamment pour les denrées standard. Par exemple, le sucre (53\%) et les conserves (60\%) sont principalement achetés chez les discounters.

Selon une enquête réalisée en 2003 par la Fédération du commerce de détail Hauptverband des Deutschen Einzelhandels (HDE), 58 \% des personnes interrogées effectuent fréquemment leurs achats alimentaires dans des magasins discount, $51 \%$ dans des supermarchés et $28 \%$ dans des magasins spécialisés (boulangerie, boucherie...). A l'inverse, seulement $4 \%$ des femmes (chargées de cette activité en Allemagne comme en France) disent ne jamais faire leurs courses alimentaires dans un magasin discount. Parallèlement, dans une autre étude de la fédération HDE réalisée en 2003, 58 \% des sondés reconnaissent avoir des habitudes d'achat dans plusieurs magasins et effectuer des comparaisons de prix, contre $45 \%$ qui disent être fidèles au même magasin. En Allemagne comme en France, la clientèle se montre de plus en plus volage.

L'un des facteurs déterminants mis en évidence est la proximité. Bien que le critère prix soit particulièrement significatif, les enseignes doivent se situer plus ou moins sur le trajet des consommateurs : domicile - école des enfants - travail. La fidélité à une enseigne est moins importante que les prix et, surtout, la praticité. C'est pourquoi l'un des axes de l'action des discounters, par le biais de la communication des offres spéciales, est d'obliger le consommateur à augmenter la fréquence de ses visites pour profiter des promotions. En conséquence, la multiplication des magasins sous une même enseigne est un support important du développement: une étude de l'institut GfK portant sur l'optimisation du potentiel d'Aldi dans les zones d'habitation en 2002 montre que $75 \%$ des ménages allemands sont à moins de dix minutes en voiture d'un magasin Aldi. Autrement dit : un magasin Aldi se trouve sur le trajet de chaque ménage...

Or ces facteurs de succès peuvent également se transformer en obstacles. D'abord, l'extension de la gamme des produits va à l'encontre de l'impératif de simplicité : ne proposer que l'essentiel, limiter le choix des consommateurs. Ensuite, le maillage dense des enseignes de magasins discount risque à terme de constituer un frein à leur croissance : d'une part, il reste peu de lieux inoccupés sur le territoire allemand, d'autre part, cela induit un sérieux risque de cannibalisation des ventes entre les magasins d'une même enseigne. La forte croissance des budgets publicitaires d'Aldi et Lidl, en tête des annonceurs, révèle combien la concurrence est vive sur le marché du discount : de janvier à mai, Aldi a investi 96 millions $€$ bruts dans la publicité (+45\%) ; Lidl, son challenger, dépensait 128 millions € (+25\% ; données Nielsen Media Research).

D'où la nécessité pour les discounters allemands de développer les implantations à l'étranger. Leur réussite en France est incontestable. Dans un contexte économique tendu, la baisse du pouvoir d'achat se fait également sentir en France, et les ménages n'hésitent plus à acheter certains produits standard à bas prix. Ainsi, la part de marché des discounters alimentaires en France est passée de $9 \%$ à 12,4\% ces trois dernières années. En réaction à cette offensive des discounters allemands, les grands distributeurs alimentaires français ont organisé la riposte en créant eux aussi des enseignes discount, comme Ed (groupe Carrefour) ou Leader Price (groupe Casino). Pour que cette initiative se transforme en un succès avéré, il leur a fallu adopter la logistique et la communication sous-jacentes à ce mode particulier de distribution alimentaire...

LE PHÉNOMĖNE DISCOUNT EST PARTICULIÈREMENT BIEN INSTALLÉ en Allemagne. La stratégie claire des enseignes (offre restreinte de produits, afin de limiter les contraintes et de négocier les prix sur de gros volumes) et la logistique propre mise en place (achats centralisés et plateforme d'approvisionnement des maga-
Des clients plus fidèles à l'offre discount qu'aux enseignes

Proximité : un argument-clé

Les atouts se transforment en frein de la croissance...

... et forcent l'expansion à l'international 
sins régionalisées) leur assurent une croissance financière indéniable, d'autant que leur modèle économique coïncide avec une demande avivée par un pouvoir d'achat en stagnation depuis quelques années. Le paysage de la distribution alimentaire s'en est trouvé considérablement modifié outre-Rhin : si le commerce traditionnel de taille moyenne est en recul, ce sont essentiellement les supermarchés qui ont pâti de la montée en puissance du discount, voyant tomber leur part de marché en dessous de celle de leurs concurrents. A l'inverse, le petit commerce de détail a pu consolider ses positions, voire les accroître légèrement, en proposant une offre complémentaire. Car dans un contexte où les crises alimentaires se multiplient, il a su mettre en avant un argument de choix : se différencier des discounters par la qualité des produits, en médiatisant la traçabilité et les labels qualité par exemple, ainsi que sur les services et conseils apportés aux consommateurs. Une stratégie qui a visiblement séduit une population désorientée et en quête de sécurité alimentaire.

Mais sur le territoire allemand, l'expansion du discount alimentaire pourrait atteindre ses limites dans un avenir proche : la très rapide multiplication des lieux de vente au cours des trois dernières années laisse entrevoir les prémisses d'une saturation du marché, avec les risques inhérents de cannibalisme. Dès lors, les discounters allemands se redéploient sur le sol européen, mettant à profit la nouvelle transparence des prix liée à la monnaie commune, et portés par une conjoncture peu florissante dans une zone euro où les consommateurs sont de plus en plus attentifs aux prix et ne s'en cachent plus. Né dans la pénurie d'après-guerre et ancré à l'origine dans les valeurs socio-culturelles allemandes, le phénomène discount s'étend aujourd'hui à l'Europe entière. «En fonction du produit recherché, le consommateur peut être client à la fois du réseau hard discount, des hypermarchés et, pourquoi pas, de Fauchon », constate J.-P. Loisel, directeur du département consommation au Crédoc, à propos des Français (Challenges, janvier 2004). Le même constat vaut pour les Allemands. Bas prix, généraliste et luxe : I'harmonisation des comportements dans la diversification des lieux d'achat est aujourd'hui un phénomène européen.

\section{Indications bibliographiques}

APPEL Cornelia, Schipperges Michael, « Les Allemands, l'euro et la mondialisation », Regards sur l'économie allemande, $n^{\circ}$ 59/2002

«Branchenreport Wirtschaft », Journal der IHK Saarland, n 06/2003

BRANDES Dieter, Die 11 Geheimnisse des ALDI-Erfolgs, Campus Verlag, 2003

Centrale MaRKeting-Gesellschaft der deUtschen AgRaRWIRTSChaft (www.cma.de)

GeSEllschaft FÜR Konsumforschung, «Ergebnisse einer GfK-Studie zu Konsumtrends in sechs europäischen Ländern », 5/03/2004, « Discounting wird zum Prinzip im Handel » et «Studie zu den Folgen des Angebots von Frischfleisch durch Discounter », 17/04/2003 (www.gfk.de)

HauptVerband des Deutschen Einzelhandels, « Allgemeine Einkaufsgewohnheiten », Mediaedge, 2004 et « Einkaufsstättenpräferenzen für Lebensmittel und Getränke", Kommunikations-Analyse, 2002 (www.hde.de)

Zentrale Markt- und Preisberichtstelle für ERZeugnisse der LAND-, Forst- UND ERNÄHRUNGSWIRTSCHAFT, Nachfrage privater Haushalte nach Nahrungsmitteln 2003, 01/2004 (www.zmp.de) 